\title{
First Swiss 3Rs Day - Implementing the 3Rs to Improve Animal Welfare and Research Quality
}

doi:10.14573/altex.2003021

On the occasion of the $60^{\text {th }}$ anniversary of the 3 Rs principle described by Russell \& Burch in their book The Principles of Humane Experimental Technique, the Swiss 3R Competence Centre organized the first Swiss 3Rs Day in the Swiss capital Bern on September 2, 2019 ${ }^{1}$. The 186 participants received up-to-date information on progress achieved in the implementation of the 3Rs principle and learned about challenges, practical solutions, and success stories from Switzerland and abroad. The program ${ }^{2}$ included dedicated sessions on each of the 3Rs with invited lectures, oral abstract presentations, poster sessions, a debate session, and a keynote lecture.

\section{Swiss 3Rs Award}

Following the opening of the conference, Kristin Schirmer and Melanie Fischer from the Federal Institute of Aquatic Science and Technology Eawag received the first 3RCC 3Rs Award in recognition of their work in the area of replacement. The researchers developed a cell line assay that reliably predicts fish acute toxicity for a wide range of chemicals and water samples ${ }^{3}$. In her award lecture, Kristin Schirmer outlined how her team de-

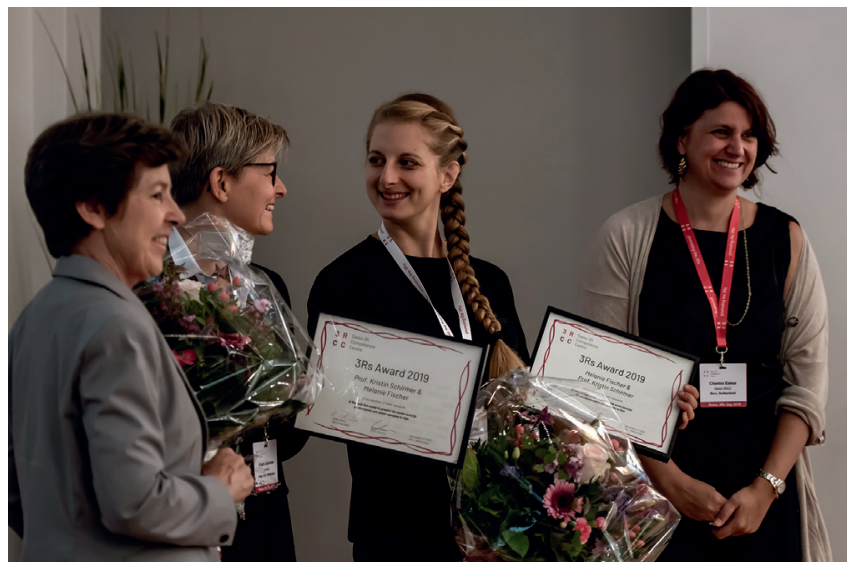

3RCC 3Rs Award

from left to right: 3RCC President Kathy Riklin; award winners: Kristin Schirmer \& Melanie Fischer, Eawag; 3RCC Executive Director Chantra Eskes veloped the assay based on the gill cell line RTgill-W1, which originates from rainbow trout Oncorhynchus mykiss. The safety test to assess environmental hazards will replace the fish acute toxicity test, where researchers expose fish to chemicals or water samples for four days and record their deaths. Given its frequent and global use, the estimated number of fish sacrificed per year in this test is in the millions. The new assay has been thoroughly tested and validated, first by the Schirmer group and subsequently in an international round-robin study with laboratories from industry and academia to demonstrate the robustness of the RTgill-W1 cell line assay and its accurate performance when carried out by operators in different laboratory settings. Thanks to these efforts, the International Organization for Standardization (ISO) has approved the assay as the first international standard based on a fish cell line. The group is now developing a serum-free culture medium to replace the fetal calf serum currently used to grow the cells.

\section{Replacement}

Strategies to replace animal models were most strongly represented at the 3Rs Day with invited lectures, oral abstract presentations as well as a debate on non-animal derived antibodies, and a keynote lecture on alternatives in education and training. Session Chair Thomas Singer from Roche outlined the efforts his company is undertaking to develop and validate alternative methods and novel technologies to reduce animal testing in drug development. Approaches include the use of innovative in silico modeling and simulation, advanced analytics, and human derived cell-based organ-on-a-chip models that minimize the number of experiments and animal studies, while improving predictivity of preclinical drug development programs for clinical trials. The company has also constructed a new in vivo facility designed to provide optimized housing for animals. Co-chair Matthias Lütolf from the Laboratory of Stem Cell Bioengineering at EPFL explained how his group develops stem cell microenvironments to improve organoid reproducibility and function ${ }^{4}$. Organoids form through poorly understood morphogenetic processes in which initially homogeneous ensembles of stem cells spontaneously self-organize in suspension or within permissive three-dimensional extracellular matrices. Yet, the absence of

\footnotetext{
1 https://www.swiss3rsday.com/

2 https://www.swiss3rsday.com/programme

3 https://doi.org/10.1093/toxsci/kfz057

4 https://www.sciencedirect.com/science/article/pii/S1934590919302097?via\%3Dihub
} 
virtually any predefined patterning influences, such as morphogen gradients or mechanical cues, results in extensive heterogeneity. The current mismatch in shape, size and lifespan between native organs and their in vitro counterparts hinders the latter's wider applicability. In his talk, Lütolf discussed the potential of next-generation organoids that his group assembles by guiding cell-intrinsic self-patterning through engineered stem cell microenvironments, opening up exciting prospects for drug discovery and regenerative medicine.

Following the invited lectures, researchers presented results of some promising approaches. Laura Suter-Dick from the University of Applied Sciences and Arts Northwestern Switzerland presented her group's in vitro human Alzheimer's disease model, which successfully incorporates key features of the disease, namely accumulation of amyloid beta plaques and formation of neurofibrillary tangles and neuro-inflammation, which lead to a loss of neurons and synaptic connections. This model could further help elucidate pathological mechanisms of the disease and accelerate drug discovery by identifying new targets and therapies. Soheila Zeinali from the Organs-on-Chip Technologies Laboratory of the University of Bern helped develop a dynamic in vitro model of human microvasculature that allows researchers to study the effect of physiological and pathological cyclic stretch on isolated human microvessels. The platform allows the study of the mechanotransduction mechanism and vascular remodeling in more detail than in vivo studies. Fabrice Müller from the ETH Zurich explained how his group developed a high-content imaging approach to simultaneously quantify mechanism-based markers for non-alcoholic fatty liver disease in metabolically competent human liver cells and extrapolate human oral equivalent doses for molecular responses. Their work will help researchers to evaluate toxicological hazards of new food-related chemicals without animal testing. The next steps are to establish a predictive model and screen food-related chemicals for their risk to induce the disease, which affects about $30 \%$ of the adult population.

Following the presentation session, Andreas Plückthun from the University of Zurich and Pierre Cosson from the University of Geneva debated the challenges associated with non-animal-derived affinity-reagent antibodies. While the technology to produce recombinant human antibodies through biotechnological methods has existed for over two decades, and all antibodies used for human therapy - those with the highest quality requirements - are produced with this technology, most researchers in biology and biomedicine still use antibodies from immunized animals, typically mice and rabbits. According to the two experts, the main reasons researchers do not use non-animal antibodies is because they are not aware of what is possible today, and traditional manufacturers do not yet offer them. Recombinant antibodies not only reduce the use of animals, they also generate more reproducible results. Only by using a recombinant antibody can two different experimenters be certain that they are using exactly the same reagent and meaningfully compare results.
The experts urged researchers still using traditional antibodies to switch to recombinant antibodies. They described available tools and services that allow generating recombinant antibodies and next-generation binding reagents against specific targets, sequencing existing monoclonal antibodies in order to convert them into recombinant reagents, and identifying existing recombinant antibodies. During the discussion, the presenters highlighted the challenges around today's reagent antibodies and offered possible solutions, including next-generation reagents that surpass what is possible with traditional antibodies ${ }^{5}$.

During his keynote lecture, Nick Jukes from InterNICHE, United Kingdom, presented best practice and alternatives in education and training for anatomy, physiology, pharmacology, clinical skills and surgery. He outlined innovative and humane methods that are available to replace the use of animals, enhance the acquisition of knowledge, skills and attitudes, and respond to demands of students, trainees and campaigners. Alternatives include non-animal tools such as advanced synthetic cadavers and training mannequins, 3D printed materials, software and virtual reality. Jukes also presented alternative approaches such as student self-experimentation, client donation programs for ethically sourced animal cadavers, case-based clinical learning opportunities with patients, and ethical fieldwork. Jukes dispelled some of the myths and misunderstandings concerning animal use and alternatives in education and training, arguing that a re-definition of the 3 Rs is necessary.

\section{Reduction}

Hanno Würbel from the University of Bern chaired the session on reduction. He warned about the alarmingly low reproducibility of animal research and identified potential causes, including poor scientific rigor, low statistical power, analytical flexibility, and publication bias. Würbel explained that the reproducibility of a result is also a function of its external validity. Unless results are robust against common sources of variation between independent replicate studies, they will not be reproducible. In animal research, effects of experimental treatments usually vary depending on the phenotype of the animals. Because the phenotype depends also on the environment of the animals, small differences in the environment between replicated studies can produce conflicting results. Therefore, he proposes to introduce systematic variation through systematic heterogenization rather than employing a more rigorous standardization to improve reproducibility. Based on theory, simulations with existing preclinical animal data, and experimental results, he showed how heterogenization of study populations improves the external validity and, therefore, reproducibility of results without a need for larger sample sizes. He concluded that using more heterogeneous study samples is crucial to avoid wasting animals for inconclusive research.

In the oral abstract presentations, Daniel Razansky from the University of Zurich and ETH Zurich described the promising potential of multi-spectral optoacoustic tomography (MSOT)

\footnotetext{
5 https://www.unige.ch/medecine/antibodies/

https://www.bioc.uzh.ch/de/forschung/core-facilities/high-throughput-binder-selection-platform/
} 


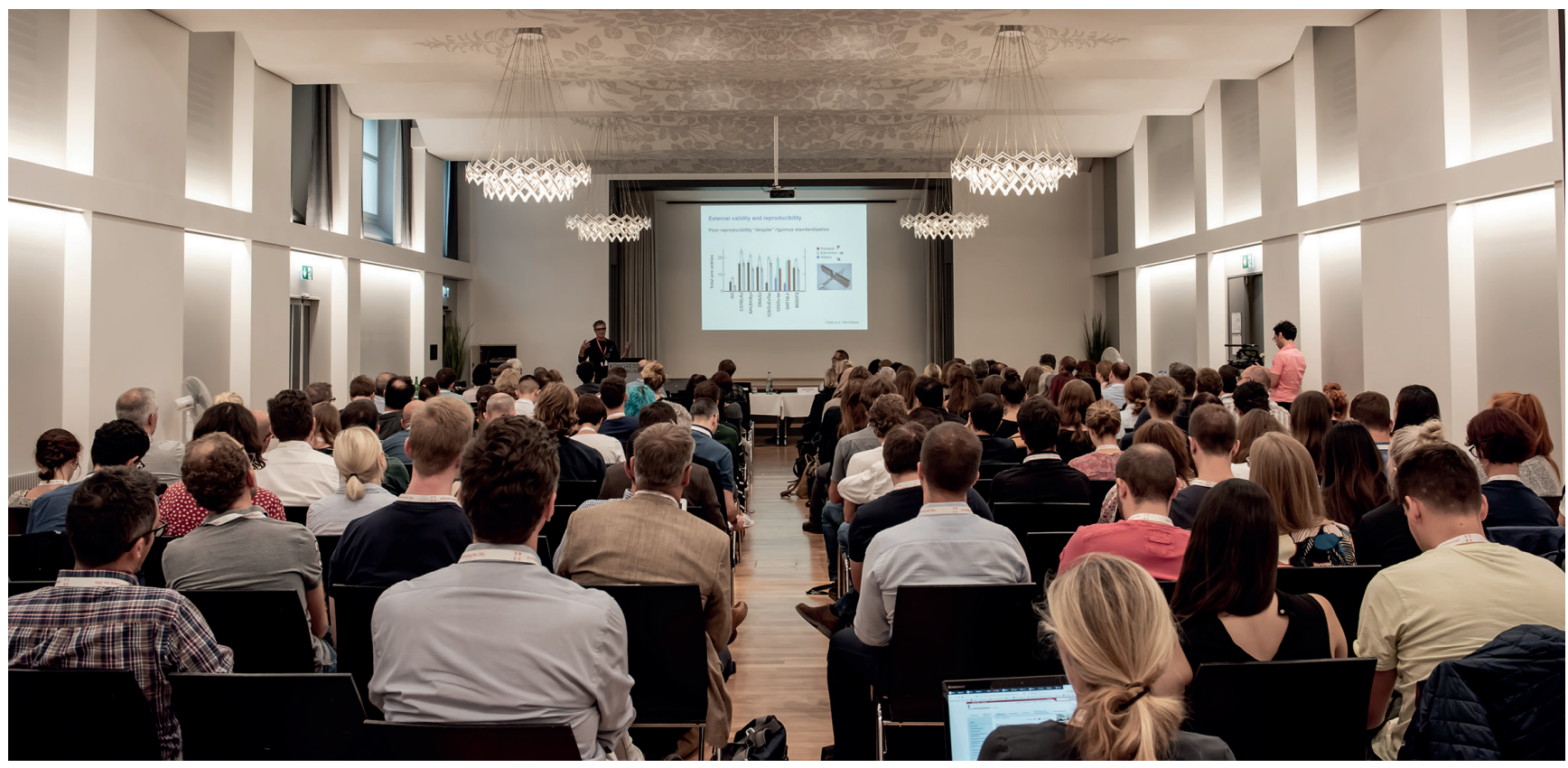

technology, which allows entirely non-invasive longitudinal observations on the same animal at penetration and spatiotemporal scales not covered by optical microscopy methods. The development of this novel functional and molecular imaging modality has enabled unprecedented insights into in vivo biological dynamics and molecular processes. Romain-Daniel Gosselin from the CHUV in Lausanne urged researchers to use sufficiently powered statistical design to reduce the number of animals. Although this may seem contradictory at first, Gosselin explained that parameters other than sample size define statistical power. He offered a brief overview of ways to optimize statistical design and successfully reduce the number of animals in the longer term. Annemarie Lang from the Charité in Berlin presented AniMatch ${ }^{6}$, an innovative web-based platform that allows scientists to register and publish or search for offers to connect with other scientists and share organs and tissues of killed animals to facilitate their use by different researchers. With this tool the researchers address the need for practical solutions that help reduce the number of animals used in experiments and, in particular, also those that are bred for the collection of tissue and organs.

\section{Refinement}

Refinement session Chair Paulin Jirkof from the University of Zurich outlined the potential that a "culture of care" has to improve animal welfare. The term indicates an organizational commitment to improve animal welfare, scientific quality, care of the staff as well as transparency for stakeholders. Simply having animal facilities and resources that meet the legal requirements is not enough to ensure that best practice animal welfare, care and use will follow, Jirkof explained. All those involved in the care and use of animals should be committed to the 3Rs principle and demonstrate a caring and respectful attitude towards the animals bred or used for scientific purposes. This includes an individual's positive and proactive mind-set and approach to animal welfare and humane science. During her talk, she presented key criteria of a culture of care and discussed how they can facilitate refinement initiatives and improve animal welfare.

Viktoria Schreiner from the University of Basel presented her research on the development of a sustained-release depot formulation of buprenorphine based on biodegradable microparticles, which can be used for pain relief in experimental animals. Achieved serum and brain concentrations of the drug as well as thermal sensitivity assays show that the depot formulation offers analgesic efficacy for at least 48 hours, which is significantly longer than when using the marketed standard formulation. Thom Ghent from the University of Zurich presented a report on the 3R symposium on euthanasia alternatives to carbon dioxide. The goal of the event, organized by the Swiss Food Safety and Veterinary Office, was to define a strategy to identify research priorities that ultimately will lead to the identification and implementation of stunning and killing methods that are more humane than the current practice of carbon dioxide exposure. Experts identified a need for consistent use of terminology across studies as well as consensus and validation of behavioral testing of aversion and the distress caused by controlled atmospheric stunning. During the symposium, experts discussed research strategies that would target mice, rats, pigs and poultry as well as the respective idiosyncrasies of each species. Mattea Durst from the University Hospital in Zurich investigated the severity, monitored pain and decreased wellbeing, and assessed the effect of analgesia in the acute cerulean-induced pancreatitis mouse model. Preliminary re-

6 https://www.animatch.eu/ 
sults indicate that cerulean injections may be acutely painful to mice and that a low level of pain is due to inflammation of the pancreas. Anne Zintzsch from the University of Giessen in Germany described the challenges researchers face in consistent severity classification. Although consistent severity assessment and classification of procedures are essential for the ethical review process and are an indispensable part of planning, refining and evaluating animal experiments, the classification is still insufficiently harmonized within EU countries, and experts often evaluate animal procedures very differently. She has produced an overview of classifications of experimental techniques and procedures and of severity classification of genetically altered animals ${ }^{7}$ that can help compare animal statistics from different countries.

The 3RCC also recognized the presentations of two young researchers at its 3Rs Day. André dos Santos Rocha from the University of Geneva won the 3RCC Poster Award for his presentation on a refined rabbit model in respiratory medicine, while Victoria Schreiner from the University of Basel received the 3RCC Oral Award for her presentation on the sustained release formulation of a painkiller used in mice. 3RCC Director Chantra Eskes closed the event with a short presentation on the center's main activities, which included the setting up of a successful funding program, the development of an education strategy at bachelor level, the organization of a conference dedicated to the $3 \mathrm{Rs}$, and communication measures that help promote the implementation of the 3 Rs principle among researchers. The Federation of Swiss Cantonal Veterinary Officers (VSKT) accredited the Swiss 3Rs Day as one day of continuing education for study directors and experimenters. The $2^{\text {nd }}$ Swiss $3 R$ s Day is scheduled to take place on September 3, 2020.

\section{Chantal Britt}

Swiss 3RCC, Bern, Switzerland

7 https://norecopa.no/severity

\section{Meeting Report}

\section{One Year Charité 3R - Results and Perspectives}

doi:10.14573/altex.2002251

One year after its official inauguration, Charite $3^{\mathrm{R} 1}$ held its first scientific symposium on December 17, 2019. Over 120 participants gathered in the full auditorium to hear about the mission and goals of the center that was founded by Charité Universitätsmedizin Berlin in 2018. The symposium also functioned as the "onboarding" of the new Scientific Advisory Board (SAB), which convened for its first working meeting the following day.

The Charité dean Axel Pries opened the scientific program of the symposium. He offered his perspective on how Charite as one of the largest university hospitals in Europe could contribute to higher quality of animal experiments and better translation by initiating a 3R center. Stefan Hippenstiel, the speaker of Charité $3^{\mathrm{R}}$, explained how this vision was put into practice during the last year. He presented examples ranging from communication and outreach, training the new generation of scientists, and funding projects leading to the development of alternative methods that can generate excellent research. He empha- sized in particular the number of projects that have been funded to generate tools that either reduce or completely replace animal experiments, or improve the living conditions of animals. In this respect, all 3Rs are equally supported by the center.

The next talks dealt with achievements of the 3Rs and 3R centers. Tom Bengtsen, head of the secretariat of the Danish $3 R$ Center and SAB member of Charite $3^{R}$, focused on the communication of the numbers of animals used in experiments to local authorities and other interested parties. He explained the benefit of a transparent and active communication strategy that starts at the level of primary education. Peter Kunzmann, who joins the Charite $3^{R}$ SAB as professor of animal ethics at the Institute for Animal Hygiene, Animal Welfare and Farm Animal Ethology at the University of Veterinary Medicine Hannover, presented the ethical perspective on whether animal experimentation is justified. Referring to the European Directive 2010/63/EU², the Nuffield Council on Bioethics Annual Report of $2005^{3}$ and other

\footnotetext{
1 www.charite3r.charite.de

2 https://eur-lex.europa.eu/legal-content/EN/TXT/PDF/?uri=CELEX:32010L0063\&from=DE

3 https://www.nuffieldbioethics.org/assets/pdfs/NCOB-2005-Annual-Report.pdf
} 